\title{
"Decision-making" in conflicts of wild chimpanzees (Pan troglodytes): an extension of the Relational Model
}

Published online: 18 October 2003

(C) Springer-Verlag 2003

\section{Behav Ecol Sociobiol (2003) 54:491-504}

In Figure 5, in the second graph from the top, the name of the independent variable ( $\mathrm{x}$-axis) is wrong. Instead of 'likelihood to', it should be 'likelihood of winning'. The correct figure is given here.

Fig. 5 Schematic illustration modelling the factors affecting two fighting strategies in Taï chimpanzees. The illustration follows our proposed order of the "decision-making" process. How the costs or benefits considered in each step affect the variation in initiation frequencies of conflicts is shown, and also how social costs affect the variation in the frequency of reduction of social costs. The positive or negative slopes of the graphs are based on the observed frequencies of conflicts in Taï chimpanzees (see Fig. 1, 2 ,3, and 4), using a straight line for simplicity. Two strategies were found: strategy A is used by dominant initiators (thick white arrow) whereas strategy B is used by subordinate initiators (thin black arrow). The outcome of the two strategies is represented by the net benefit in relation to the relative initiation frequency of conflicts for dominant $(A$, white $)$ or subordinate $(B$, black) initiators. The outcomes of both strategies are presented in a simplified form (the most likely outcome) and are shown together in one graph to clarify the trade-off used by subordinate conflict partners: subordinate initiators trade their lower frequency of winning a conflict for a greater positive net benefit, when they are winning, and a smaller negative net benefit, when they are losing, compared to dominant initiators (see Discussion)

The online version of the original article can be found at http://dx.doi.org/10.1007/s00265-003-0654-8

R. M. Wittig $(\bowtie) \cdot$ C. Boesch

Department of Primatology,

Max Planck Institute for Evolutionary Anthropology,

Deutscher Platz 6, 04103 Leipzig, Germany

e-mail: wittig@eva.mpg.de

Tel.: +49-341-3550202

Fax: +49-341-3550299
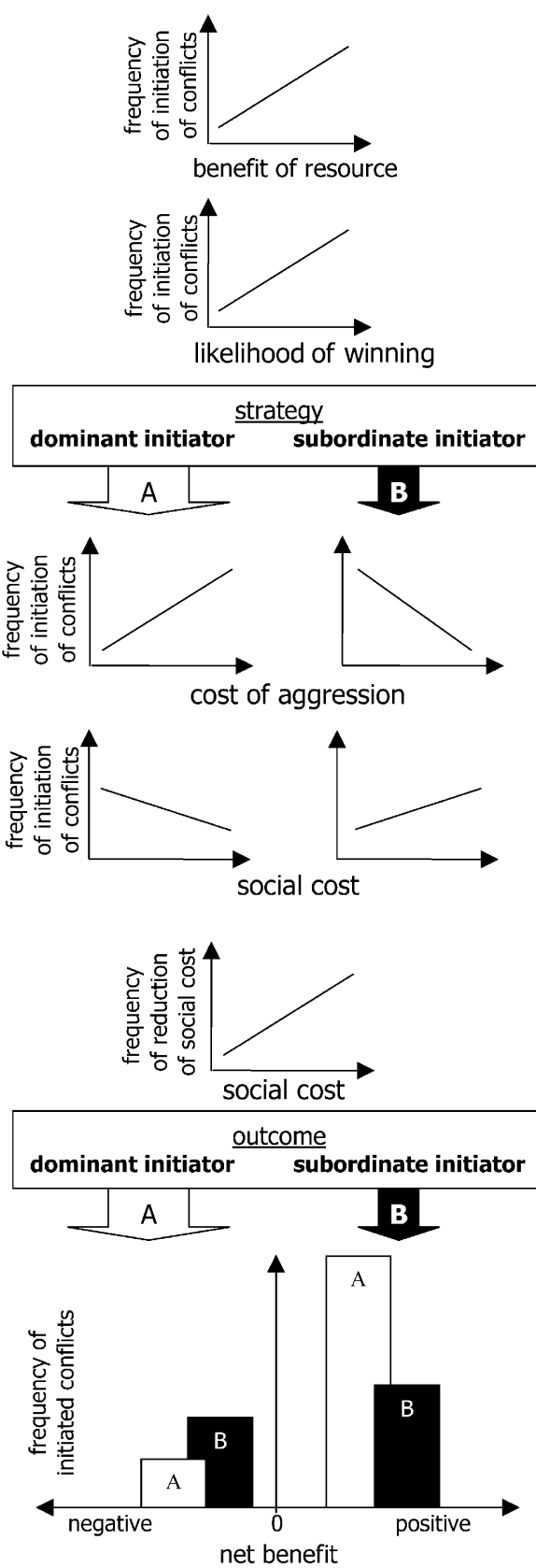